\title{
ROBUST LATERAL BLENDED-WING-BODY AIRCRAFT FEEDBACK CONTROL DESIGN USING A PARAMETERIZED LFR MODEL AND DGK-ITERATION
}

\section{A. Schirrer, C. Westermayer, M. Hemedi, and M. Kozek}

\author{
Institute of Mechanics and Mechatronics \\ Vienna University of Technology \\ 8 Wiedner Hauptstr., Vienna 1040, Austria
}

\begin{abstract}
This paper shows control design results, performance, and limitations of robust lateral control law designs based on the DGK-iteration mixed- $\mu$ synthesis procedure for a large, flexible blended wing body (BWB) passenger aircraft. The aircraft dynamics is preshaped by a low-complexity inner loop control law providing stabilization, basic response shaping, and flexible mode damping. The $\mu$ controllers are designed to further improve vibration damping of the main flexible modes by exploiting the structure of the arising significant parameter-dependent plant variations. This is achieved by utilizing parameterized Linear Fractional Representations (LFR) of the aircraft rigid and flexible dynamics. Designs with various levels of LFR complexity are carried out and discussed, showing the achieved performance improvement over the initial controller and their robustness and complexity properties.
\end{abstract}

\section{INTRODUCTION}

Flexible aircraft control is by now a widely studied task (see, for example, $[1-5]$ ), driven by potential weight savings and thus potentially increased fuel efficiency. One particularly interesting concept in civil aviation are BWB configurations which bear an additional potential of reduced fuel consumption per passenger, but they also pose new challenges in multiobjective control design [6]: potential (cross-)coupling of longitudinal and lateral motion (and low-frequency flexible modes), possible open-loop instability, as well as high performance demands in loads alleviation, vibration reduction, and maneuver shaping. 
Flight and structural control laws are commonly built using robust control design methods to ensure satisfactory control performance also in the presence of plant uncertainties. The DK-iteration and, more recently, the DGK-iteration or mixed- $\mu$-synthesis are well-known design tools to generate such control laws $[7-9]$.

This paper presents a state-of-the-art flight control design for a novel application: control of the lateral dynamics of a large, flexible BWB passenger aircraft. A multitude of stringent constraints and goals are given in the time and frequency domain. An initial controller is designed using robust modal control design $[10,11]$ to achieve some of the goals most closely related to eigenstructure assignment. Based on the preshaped plant, a parameterized high-accuracy LFR is built which serves as basis for robust feedback control design by DGKiteration. Due to high-dimensional parameter dependency and nontight bounds in current $\mu$ analysis tools, this synthesis task faces computational difficulties given today's workstation computing performance and numeric properties of the algorithms. Thus, the ways to reduce design complexity and improve resulting robust control performance are tested and assessed in terms of performance, robustness, tractability, and problem size. A high-accuracy parametric LFR as well as various simplified LFR formulations are utilized in subsequent design attempts.

A general integrated methodology for multiobjective robust control design has been presented in [12]. Previous, closely related studies started on a larger BWB passenger aircraft predesign model: for LQ-based lateral control designs see [13], the application of a genetic algorithm for parameter optimization of a multiobjective $\mathcal{H}_{\infty}$ DK-iteration design has been treated in [14]. Using a Youla parameterization of the feedback control loop, a convex controller synthesis for lateral BWB control has been performed in [11] with a subsequent scheduled feedforward control design in [15]. Longitudinal BWB control using LPV control concepts has been studied in [16]. The models of the currently studied BWB passenger aircraft are obtained by highly detailed modeling and are expected to yield more reliable results for control validation.

This paper is structured as follows:

- section 2 introduces the aircraft model, provides an overview on its openloop characteristics, and formulates the envisaged control goals;

- section 3 outlines the control design variants and attempts that were carried out to fulfill the control goals;

- in section 4, the final design is validated at all validation cases of the aircraft model and its performance is assessed. The main issues from a design perspective are discussed; and

- section 5 concludes the paper. 


\section{SYSTEM MODEL}

Dynamic models of a large BWB passenger aircraft predesign configuration have been developed by the authors' project partners [17]. These integrated models represent the longitudinal as well as the lateral flight mechanics of the rigidbody aircraft motion, the flexible structure mechanics, as well as the aeroelastic coupling of the rigid-body and the flexible dynamics. The Finite Element (FE) method has been utilized for structural design, panel- and computation fluid dynamics methods have been applied to obtain aerodynamic data. Compared to earlier studies [11, 13-15], the present models consider a redesigned, downsized BWB configuration.

Four relevant parameters are considered of having a major effect on the dynamic system behavior: the flight parameters airspeed and dynamic pressure as well as the two structure parameters fuel filling level and center-of-gravity $(C G)$ position. The longitudinal and lateral flight mechanics equations have been linearized about level trimmed flight conditions at fixed parameter gridpoints, and the flexible structure modes have been modeled and separated into symmetric and antisymmetric modes. Then, due to the symmetry of the aircraft configuration, the lateral dynamics is decoupled from the longitudinal dynamics for small perturbations of the flight mechanic variables (in particular, for sufficiently small perturbations of side-slip, roll, and yaw angles). In this predesign stage, no further quantification of lateral-longitudinal coupling effects has been done because the main objectives of the conducted studies target control design and loads alleviation in trimmed level flight conditions or in typical, moderate turn maneuvers.

This study only considers the lateral dynamics (comprised of lateral flight mechanics, antisymmetric flexible structure dynamics, as well as lag states) to design and validate the lateral control laws at a specific flight case corresponding to cruise flight (fixed airspeed and dynamic pressure). A set of $k=30$ linearized state space systems $\boldsymbol{P}_{i}, i=1, \ldots, k$ for various parameter values of fuel filling level and CG position is studied:

$$
\begin{aligned}
& \dot{\boldsymbol{x}}=\boldsymbol{A}_{i} \boldsymbol{x}+\boldsymbol{B}_{i} \boldsymbol{u} \\
& \boldsymbol{y}=\boldsymbol{C}_{i} \boldsymbol{x}+\boldsymbol{D}_{i} \boldsymbol{u} .
\end{aligned}
$$

The state vector $\boldsymbol{x}$ is composed of 4 flight-mechanic states (side slip angle $\beta$, roll rate $p$, yaw rate $r$, and roll angle $\phi$ ), 12 elastic states (6 structural antisymmetric modes), as well as 7 lag states. The integrator states $\psi$ (yaw angle) and $y$ (horizontal displacement) are neglected in this study. These systems are augmented by actuator and sensor dynamics.

Utilized inputs $\boldsymbol{u}$ for control design are:

- symmetric rudder deflection and rate: $u_{\mathrm{RU}}$ and $\dot{u}_{\mathrm{RU}}$; and 
- combined antisymmetric trailing edge (TE) control surface deflections and rates:

- $u_{\mathrm{TE} 12}$ and $\dot{u}_{\mathrm{TE12}}$ : middle and inner elevons (deflected equally); and

- $u_{\mathrm{TE} 3}$ and $\dot{u}_{\mathrm{TE} 3}$ : outer elevon.

The actuator dynamics $\boldsymbol{G}_{\text {act }}$ are modeled via second-order low-pass filters as a low-order approximation of physically modeled control surfaces and actuation system dynamics. These models provide both the actual surface deflections as well as their rates as outputs $\left(\left[u_{j}, \dot{u}_{j}\right]^{\mathrm{T}}=\boldsymbol{G}_{\text {act }, j} u_{\text {command }, j}\right)$.

Utilized outputs $\boldsymbol{y}$ for control design are:

(1) side slip angle $\beta$;

(2) roll angle $\phi$;

(3) roll rate $p$;

(4) yaw rate $r$; and

(5) antisymmetric wingtip acceleration signal

$$
\mathrm{Nz}_{\text {lat.law }}=\mathrm{Nz}_{\mathrm{r} . \text { wingtip }}-\mathrm{Nz}_{\text {l.wingtip }}
$$

where $\mathrm{Nz}_{\text {r.wingtip }}$ and $\mathrm{Nz}_{\text {l.wingtip }}$ are the vertical accelerations at the right and left wingtips, respectively,

which are each considered subject to time delays due to signal processing latencies (160 ms for outputs (1)-(4), $60 \mathrm{~ms}$ for output (5)), modeled via secondorder Padé approximations. Additionally, second-order low-pass Butterworth filters are applied to outputs (1)-(4). Note that output (5) acts as an effective, simplified modal sensor for the antisymmetric flexible modes. It amplifies these modes' amplitudes; however, it is not compensated for the effect of roll rate change (rigid-body motion) for simplicity. The sensor dynamics is collected into $\boldsymbol{G}_{\text {sens }}$, and the augmented system $\widetilde{\boldsymbol{P}}_{i}=\boldsymbol{G}_{\text {sens }} \boldsymbol{P}_{i} \boldsymbol{G}_{\text {act }}$ is of order 47 .

Additional exogenous input and output signals are considered for system analysis and validation: a wind gust disturbance input is modeled by an aggregated lateral wind speed input signal

$$
d=v_{\text {lat }},
$$

as well as a structure load output (a cut moment at the wing)

$$
z=\mathrm{My}_{\text {wing }} \text {. }
$$




\subsection{Open-Loop Characteristics}

The uncontrolled lateral aircraft dynamics shows a marginally stable or slowly unstable spiral mode for all considered mass cases at cruise flight conditions. Moreover, a prominent low-damped Dutch Roll (DR) mode between 0.7 and $0.9 \mathrm{rad} / \mathrm{s}$ with a damping ratio between 0.03 and 0.06 is present. Six relevant antisymmetric flexible modes are located between 10 and $50 \mathrm{rad} / \mathrm{s}$ of which the first two (at approximately 10 and $20 \mathrm{rad} / \mathrm{s}$, respectively) are considered critical for structure loads and comfort. Figure 1 shows the response from gust $v_{\text {lat }}$ to wing load $\mathrm{My}_{\text {wing }}$ for the uncontrolled aircraft at all considered mass cases, as well as the same response of the aircraft controlled by an initial robust pole placement controller which robustly stabilizes and assigns satisfactory rigidbody responses (see subsection 3.1). However, as evident in Fig. 1, the lowfrequency transfer magnitude from lateral gust to $\mathrm{My}_{\text {wing }}$ is increased as a result of stabilization (see discussion in subsection 3.1).

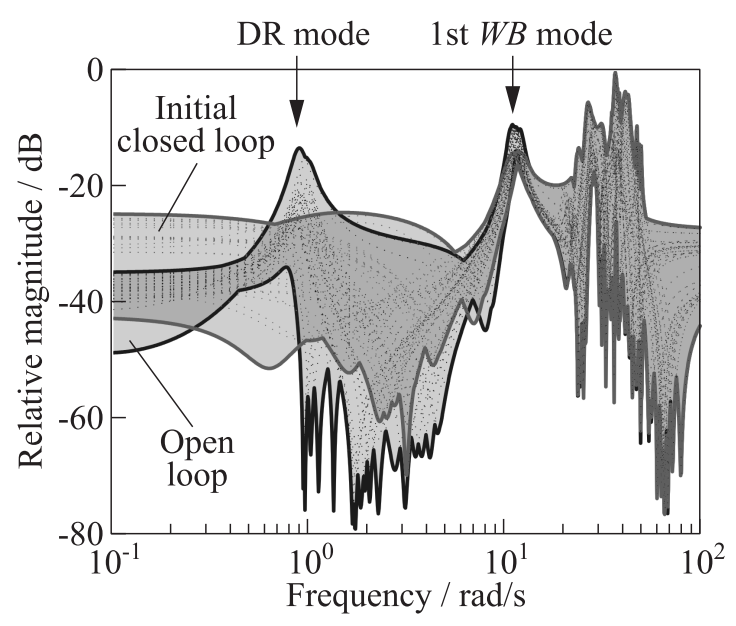

Figure 1 Bode magnitude plot of lateral wind $v_{\text {lat }}$ - wing cut moment $\mathrm{My}_{\text {wing }}$ for all mass cases (DR and wing bending (WB) modes are indicated. Black: open-loop, grey: closed loop with initial stabilizing controller, see subsection 3.1 )

\subsection{Control Goals}

The specific control goals for this lateral inner loop control design are:

(1) to stabilize the aircraft; 
(2) to obtain a high damping ratio $\zeta \geq 0.7$ of the DR mode while keeping the mode's undamped eigenfrequency unchanged;

(3) to obtain sufficiently fast real/aperiodic remaining system dynamics to fulfill rise-time requirements on roll/side slip responses in 7 and $5 \mathrm{~s}$, respectively; and

(4) to maximize damping of the first two flexible modes.

Note that in the present setting, goal 1 also includes a significant shift of the spiral mode's pole to the left which otherwise is realized by an outer (auto-)pilot control loop.

These requirements all have to be fulfilled robustly for all 30 considered parameter cases in the viewed parameter space. They will be all addressed, as far as possible, by an initial control law which is designed through robust/insensitive eigenstructure assignment. However, further improvement of the vibration damping performance (goal 4) is possible when exploiting knowledge on the parameter dependency. Thus, the focus and main control goal of this work is to improve on goal 4.

\section{CONTROL DESIGN}

\subsection{Initial Controller}

In order to efficiently fulfill the requirements on the rigid-body response of the aircraft, a robust eigenstructure assignment approach is taken. Utilizing the Robust Modal Control Toolbox (see [10]), a low-order output feedback control law is generated which robustly assigns partial eigenstructure specifications (as in $[11,18]$ ). An output feedback controller $\boldsymbol{K}_{\text {init }}$ of dynamic order 1 is obtained. The initial controller is interconnected to the aircraft system models, forming a set of preshaped plants (each of dynamic order 48). Figure 1 shows the effect of this initial control law: the aircraft is robustly stabilized, the damping ratios of the DR mode and the first flexible WB modes are increased but also an overall increase in the low-frequency magnitude of the disturbance-loads transfer becomes evident. The flight mechanic modes are assigned robustly to their desired locations and the rigid-body responses are shaped as desired. Through eigenvector projection, it is possible to reduce the first flexible mode robustly by about $-6 \mathrm{~dB}$. Figure 1 shows the improved flexible mode damping and Fig. 2 shows the correctly and robustly shaped flight dynamic response characteristics. Note that it is not possible to directly and robustly increase flexible mode damping further with this design methodology.

Further studies on the issue of the increased low-frequency disturbance-load magnitude show that this effect mainly occurs at parameter configurations far 

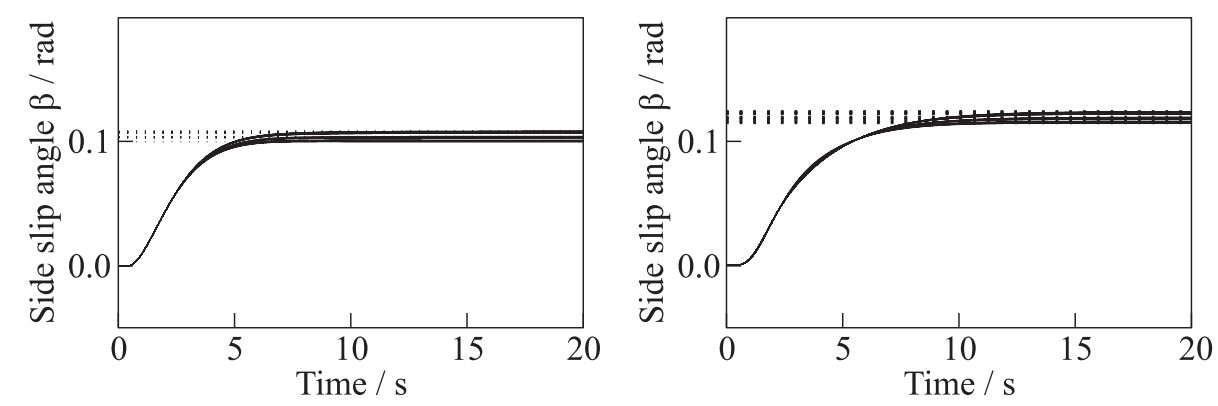

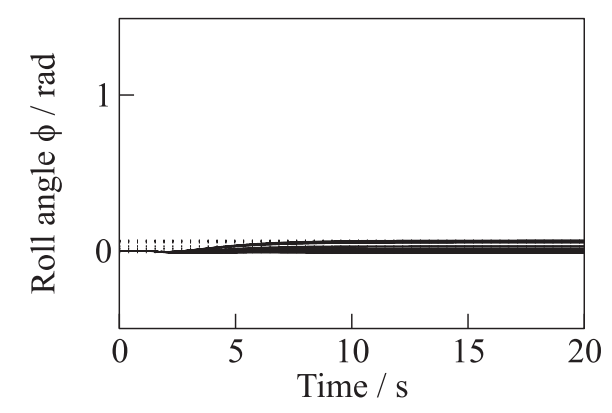

(a)

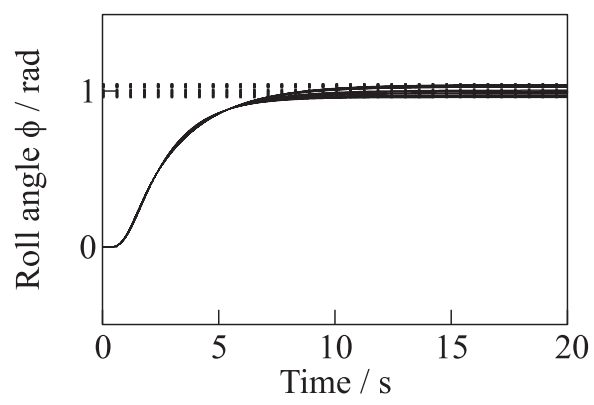

(b)

Figure 2 Step responses of the preshaped plants from rudder $(a)$ and ailerons $(b)$ to side slip and roll angles at random CG and fuel parameter values

from the design point. When solely assigning one aircraft mode to the desired location while keeping the others fixed at their open-loop locations, it is unveiled that shifting the roll mode and the flexible mode does not affect lowfrequency loads; however, both DR mode shaping as well as shifting the spiral mode are responsible to a similar degree to the observed increase in loads. Further optimization of the low-frequency disturbance behavior of the aircraft is not studied in this work, but represents an interesting area for follow-up studies.

\subsection{Linear Fractional Representation of the Parameterized, Preshaped Plants}

By exploiting the structure of the parameter dependency of the plant, the damping of the first flexible modes is attempted to be further increased without altering the other control goals (rigid-body response, stability). Therefore, an LFR description of this set of preshaped plants in the two parameters CG and fuel filling has been generated from the model grid (1)-(2) and validated by the au- 
thors' project partners analogous to the procedure in [19]. The lag states were removed for the LFR generation. A first, high-accuracy LFR has been generated which has 41 states and a $\boldsymbol{\Delta}$ block size of $40 \times 40$ (in which the two real-valued parameters are 9 and 31 times repeated, respectively). Later, due to computational difficulties with this level of complexity, a simplified parameterization has been generated which leads to a reduced-accuracy LFR with 33 states and a $13 \times 13 \Delta$ block (8 and 5 times repeated, respectively).

Figure 2 shows scaled, typical step responses (as modeled by the highaccuracy LFR) for several randomly sampled parameter values. The rigid-body response is considered satisfactorily shaped by the initial controller.

\subsection{DGK-Iteration Results}

DGK-iteration is employed with the aim to generate a robust controller that fulfills the targeted control goals: to attenuate the first and second flexible modes and thus reduce the gust-induced wing loads. For details on the involved robust control theory, fundamental definitions of linear fractional transforms (LFT) / LFR, the structured singular value $(\mu)$, robust stability (RS), robust performance (RP), or the DK- and DGK-iteration algorithms, the reader is referred to $[7-9,20]$.

The control design architecture for control design via DGK-iteration is outlined in Fig. $3 a$. The system LFR $\boldsymbol{G}_{\mathrm{LFR}}$ is augmented by the design weights $\boldsymbol{W}_{a}$, $\boldsymbol{W}_{n}, \boldsymbol{W}_{u}$, and $\boldsymbol{W}_{z}$ to obtain the augmented plant $\boldsymbol{G}_{\text {aug }}$, and $\boldsymbol{K}$ is the robust feedback LTI controller to be designed. The modeled signals are disturbance in-

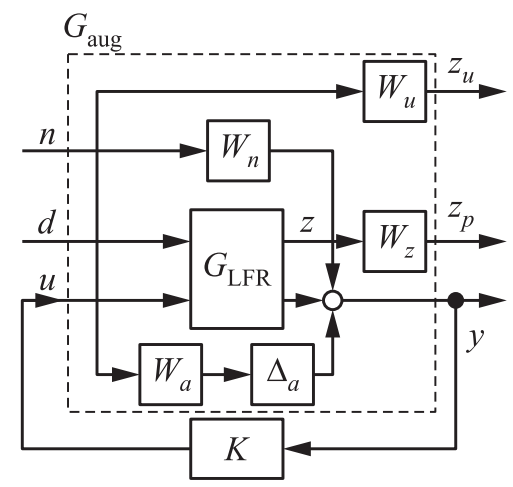

(a)

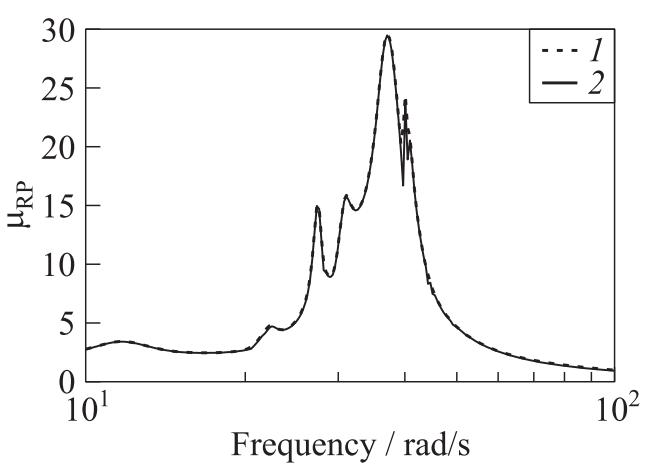

(b)

Figure 3 Design architecture for DGK-iteration $(a)$ and robust performance $\mu$ analysis results $(b): 1-\mu$ upper bound; and $2-\mu$ lower bound 
put $d=v_{\text {lat }}$, feedback control commands $\boldsymbol{u}=\left[u_{\mathrm{RU}, \mathrm{FB}}, u_{\mathrm{TE} 12, \mathrm{FB}}, u_{\mathrm{TE} 3, \mathrm{FB}}\right]^{\mathrm{T}}$, the performance outputs $\boldsymbol{z}=\left[\mathrm{My}_{\text {wing }}, \mathrm{Nz}_{\text {lat.law }}\right]^{\mathrm{T}}$, the measured outputs $\boldsymbol{y}$ $=\left[\beta, \phi, p, r, \mathrm{Nz}_{\text {lat.law }}\right]^{\mathrm{T}}$ with measurement noise $\boldsymbol{n}$, as well as the weighted output signals $\boldsymbol{z}_{u}$ and $\boldsymbol{z}_{p}$. The measurement noise weighted $\boldsymbol{W}_{n}$ and the additive uncertainty weight $\boldsymbol{W}_{a}$ serve as problem regularization terms and are chosen small and constant. The remaining weights are chosen

- to ensure well-scaled input/output magnitudes (via scaling inside $\boldsymbol{G}_{\mathrm{LFR}}$ );

- to emphasize the first and the second WB modes in the performance path $\left(\right.$ via $\left.\boldsymbol{W}_{z}\right)$; and

- to limit the control input magnitudes to the admissible input range (via $\left.\boldsymbol{W}_{u}\right)$.

\subsubsection{DGK-design attempt with high-accuracy linear fractional representations}

The results of a DGK-iteration run based on the high-accuracy LFR are shown in Fig. $3 b$.

The robust performance $\mu$ value is much larger than 1 at all considered frequencies - it is clearly evident that the closed loop fails to achieve satisfactory control performance. In further studies, it becomes evident that the bounds of the open loop robust stability $\mu$ value are very loose. This problem of convergence and the resulting conservativeness in the $D$ - and $G$-scalings yield unsatisfactory results of the design. Note that only static scalings could be utilized in DGKiteration design due to the problem size: the $\boldsymbol{\Delta}$-block contains $40 \times 40=1600$ entries. Fitting these with dynamic $G$ - and $D$-scalings inflates the controller order quickly well above 1000 which is numerically and computationally infeasible.

One common heuristics to improve mixed- $\mu$ convergence is to add small, complex uncertainties to the existing real uncertainties. This was attempted first; however, no improvement in $\mu$ bound convergence could be observed.

To overcome the encountered computational difficulties, two simplification approaches will be taken and compared in the following.

\subsubsection{DGK-design attempt with ad-hoc uncertainty model}

Based on the observation that the perturbations of the flexible mode parameters are the main source of uncertainty, an ad-hoc uncertainty parameterization is attempted (for similar attempts, see $[4,8,21]$ ). The aircraft models are close to 
a modal form [22] in which a low-damped flexible mode is represented by a $2 \times 2$ submatrix of the system matrix $\boldsymbol{A}$ :

$$
\boldsymbol{A}_{\mathrm{m} i}=\left[\begin{array}{cc}
0 & 1 \\
-\omega_{i}^{2} & -2 \zeta_{i} \omega_{i}
\end{array}\right] .
$$

By replacing the $(2,1)$ and $(2,2)$ matrix elements with real-valued uncertain parameters which are confined to the intervals occurring across the model set, an efficient uncertainty representation with a small uncertainty matrix $\boldsymbol{\Delta}$ of size $2 \times 2$ per mode is obtained. Note that no other variations in the plant are considered; hence, the uncertainty model is rather crude. The architecture shown in Fig. 3 is reused, but the plant LFR is replaced by its simplified

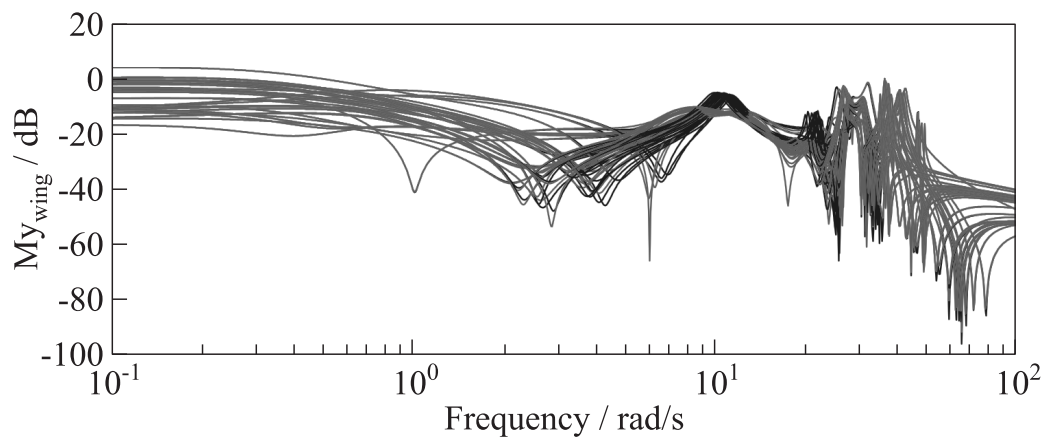

(a)

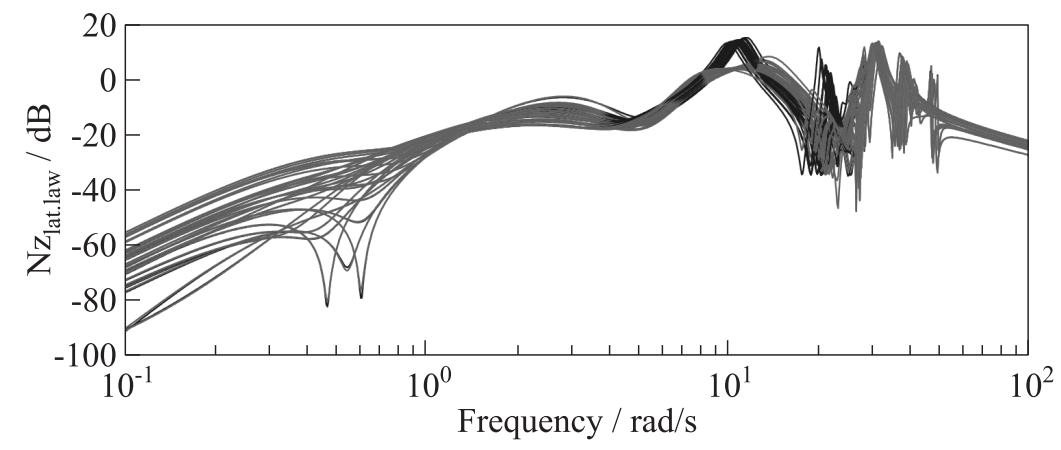

(b)

Figure 4 Bode magnitude plots of von-Karman low-pass filtered lateral wind $v_{\text {lat }}$ to wing cut moment $\mathrm{My}_{\text {wing }}$ and antisymmetric wingtip acceleration signal $\mathrm{Nz}_{\text {lat.law }}$ for all mass cases: black — preshaped design plant and grey — closed loop with robust controller obtained by DGK-iteration on a simplified design LFR 
version (with a $\boldsymbol{\Delta}$-block of $4 \times 4$ ). The achieved robust performance $\mu$ value is 2.7 .

The obtained controller is of dynamic order 117 (due to dynamic $D$ - and $G$-scalings) after few minutes of computation time on a standard office personal computer. This controller complexity is, in general, too high for implementation; so, controller order reduction is needed subsequently.

Figure 4 shows the performance singular values of the open- and closed-loop systems with the validation plants. An input turbulence model according to a one-dimensional von-Karman vertical turbulence model has been utilized to include information on the expected low-pass characteristics of turbulence excitation, assuming that a similar turbulence characteristics can be observed in a lateral direction. It is evident that for most models, the obtained controller performs well and achieves strong attenuation (about $-7 \mathrm{~dB}$ ) of the first and second flexible modes. However, in two (extremal) parameter cases, the second flexible mode of the respective validation plant is destabilized. No simple means are available to ensure stability with these plants except for enlarging the uncertainty ranges which quickly destroys the obtained nominal performance.

\subsubsection{DGK-design with reduced-accuracy linear fractional representations}

In order to obtain a computationally manageable problem size, but still to obtain a robustly stabilizing and performing control law, a reduced-accuracy parameterized LFR has been generated. The weight shapes are chosen as depicted in Fig. 5 to emphasize the control effect on the first flexible mode. After several design iterations, it became clear that the large variation of the second flexible

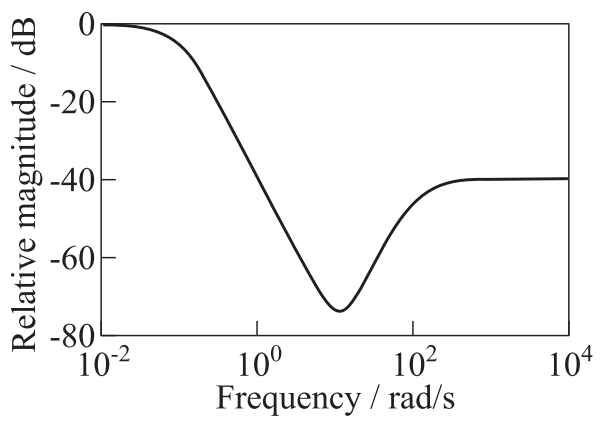

(a)

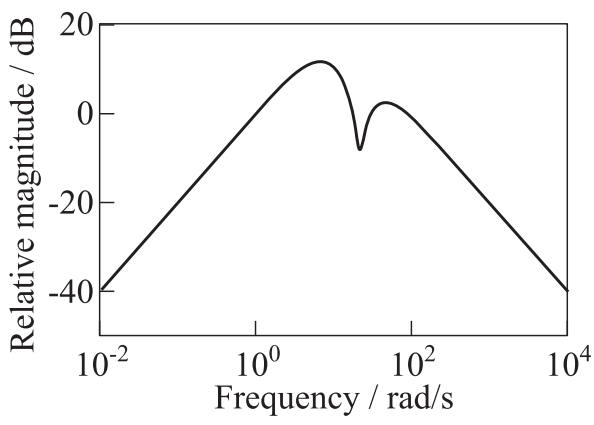

(b)

Figure 5 Weight shapes of $\boldsymbol{W}_{u}(a)$ and $\boldsymbol{W}_{z}(b)$ : the control action is focused on the first WB mode (notch in $\boldsymbol{W}_{u}$, peak in $\boldsymbol{W}_{z}$ ). Additionally, the second flexible mode must be attenuated in the performance path to obtain robust performance 

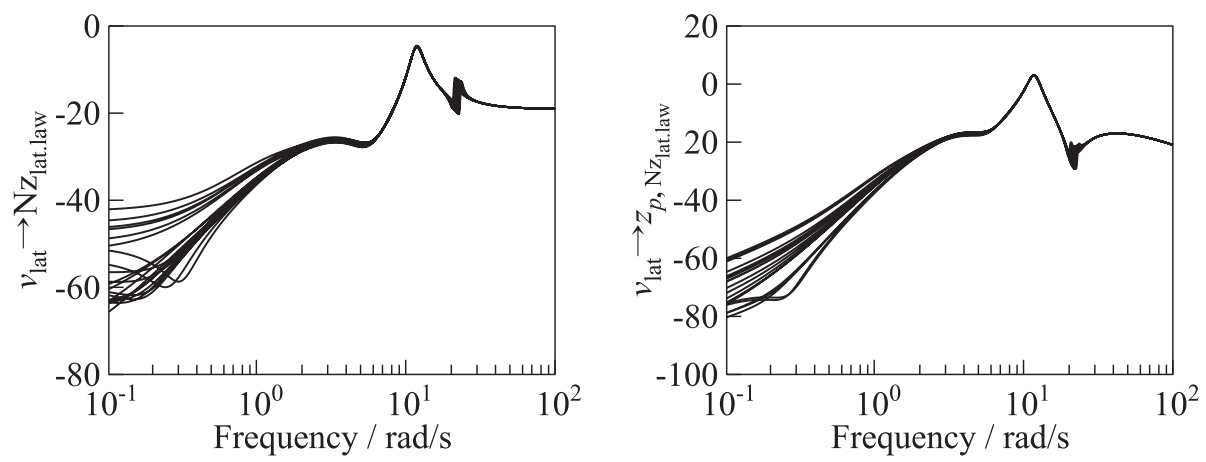

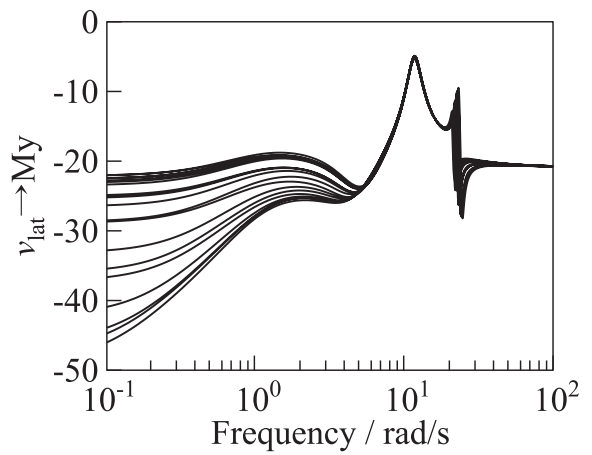

(a)

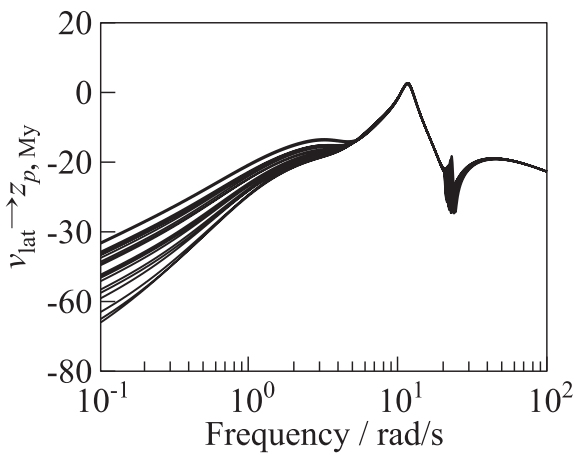

(b)

Figure 6 Scaled, unweighted $(a)$ and weighted $(b)$ magnitude plots of lateral gust (performance outputs) as modeled by the reduced-complexity LFR sampled at 20 random parameter points

mode is a limiting factor in the design - therefore, the weightings are adapted to avoid control action at the second flexible mode's frequency range.

Figure 6 shows the unweighted and the weighted performance singular values of the unweighted (scaled) LFR and of the weighted design plant, randomly sampled in the uncertain set. The effect of the chosen weightings is clearly visible - the strongly varying second mode is decreased in importance; the control design task focuses on the first flexible mode.

After the DGK-iteration run (20 iterations, $D$ - and $G$-scalings up to order 4, grid of 284 frequencies, augmented design plant $\boldsymbol{P}_{\text {aug }}$ of order 59, 135-minute computation time), a robust performance $\mu$ of 1.44 is obtained (as compared to an open-loop robust performance $\mu$ of 2.0 ), which is still larger than 1 , but, as seen in Fig. 7, the robust stability $\mu$ value is less than 1 . The figure shows also the nominal performance singular values (single weighted load performance outputs and all outputs combined) of the nominal closed loop $\boldsymbol{M}$ and thus shows 


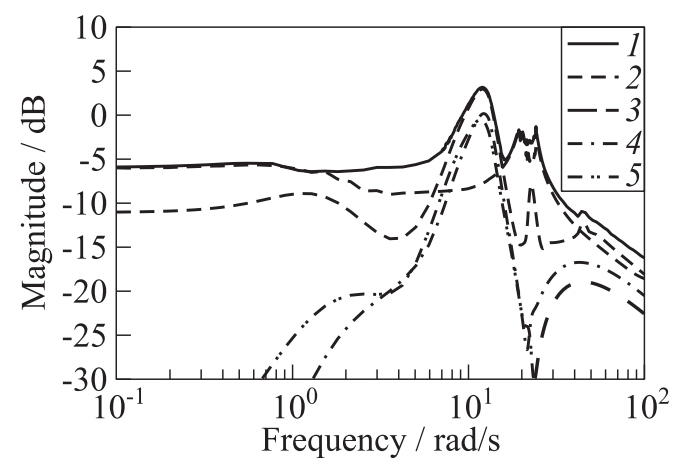

Figure 7 Nominal performance singular values and $\mu$ upper bounds for RP and $\mathrm{RS:} 1-\mu_{\mathrm{RP}} ; 2-\mu_{\mathrm{RS}} ; 3-\bar{\sigma}\left(M_{z \leftarrow v_{\mathrm{lat}}}\right) ; 4-\bar{\sigma}\left(M_{z_{p, \mathrm{Nz}} \text { lat.low }} \leftarrow v_{\text {lat }}\right) ;$ and $5-$ $\bar{\sigma}\left(M_{z_{p, \mathrm{My}} \leftarrow v_{\mathrm{lat}}}\right)$

the closed-loop system variation bounds as gap between the nominal singular values and the robust performance $\mu$ bound. The controller dynamic order is very high with 253 states. For implementation, (robust) controller order reduction must be performed (for a $\mu$-based approach, see [23]). The high-order control law can be reduced by MATLAB's reduce command [9] with the option 'ErrorType','mult' to order 30 virtually without performance loss. The underlying algorithm is a balanced stochastic model truncation (BST) via Schur's method [24].

\section{VALIDATION, PERFORMANCE, AND DISCUSSION}

\subsection{Validation of Control Performance and Robustness}

The control law obtained in subsection 3.3 is validated with all grid models (1)(2). All closed-loop systems are stable. While the $\mu$ analysis results in Fig. 7 proves Robust Stability for the utilized LFR formulation of the problem (up to LFR approximation errors), this enumeration of the set of all closed-loop systems proves Robust Stability in terms of the provided model set.

Figure 8 shows the magnitude plots of the disturbance-performance paths: the first flexible mode can robustly be reduced to $2-3 \mathrm{~dB}$ below the level provided by the initial control law. Note that this does not contradict the evident lack of Robust Performance in the LFR sense (which is based on the performance formulation according to Fig. 3). 

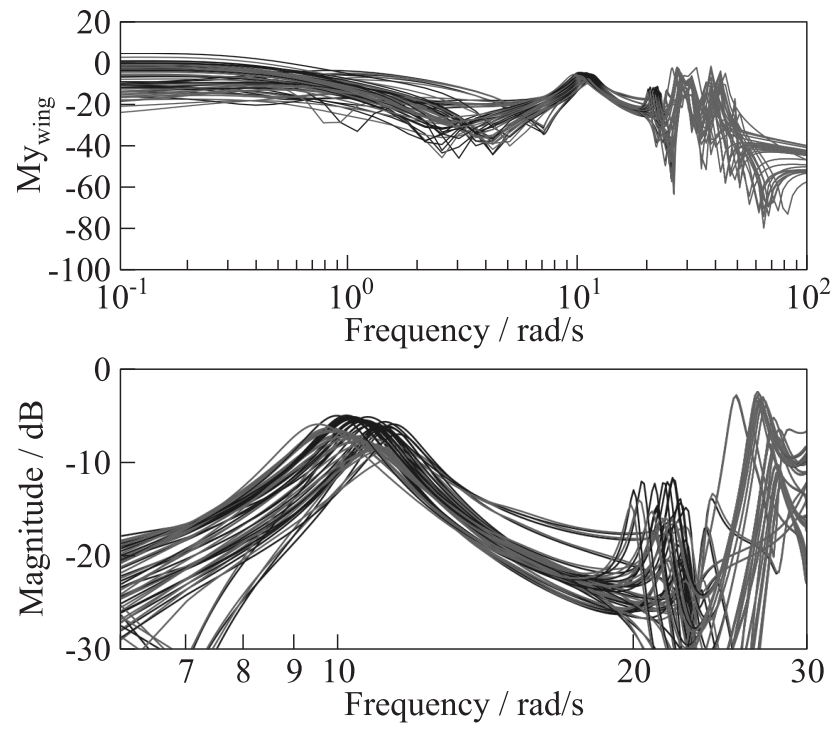

(a)
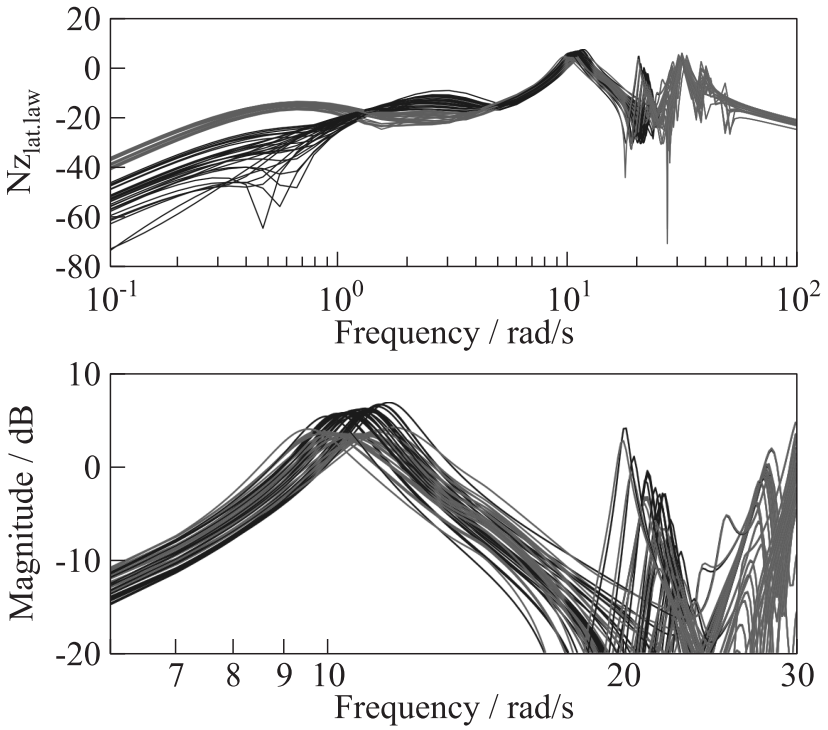

(b)

Figure 8 Bode magnitude plots of von-Karman low-pass filtered lateral wind $v_{\text {lat }}$ to the wing cut moment $\mathrm{My}_{\text {wing }}(a)$ and to the antisymmetric wingtip acceleration signal $\mathrm{Nz}_{\text {lat.law }}(b)$ for all mass cases: black curves - aircraft model with initial control law only; and grey curves - closed loop with initial controller and robust controller obtained by DGK-iteration with the reduced-accuracy design LFR 
The controller obtained by DGK-iteration does not interfere with lowfrequency roll and side-slip behavior of the BWB aircraft; so, the final closedloop responses are virtually unchanged compared to Fig. 2 and control goals 2 and 3 remain fulfilled.

\subsection{Discussion}

A highly detailed modeling process yields accurate system models for a parameter grid of relevant system parameters. For high parameterization accuracy, the obtained parameterized LFR turns out to be prohibitively complex for current $\mu$ analysis and synthesis algorithms. Several ways to solve the design task have been attempted, including well-known problem regularization techniques ("complexification" of the uncertainty description) and simplification of the LFR.

Ad-hoc uncertainty modeling yields simple LFRs and high control performance for the design plant, but it destabilizes some parameter-extremal validation plant cases in closed loop. No straightforward remedy is found without compromising control performance significantly.

Subsequently, a reduced-accuracy parameterized LFR is generated which leads to a successful, albeit computationally demanding design. The obtained control law can be reduced to order 30 without performance degradation and yields stable closed loops with all validation cases. Its performance is significantly lower than the nominal performance achieved through the ad-hoc approach but in turn, it provides an actually robust solution. Considering that significant damping is already introduced by the initial control law, it is plausible that further improvement comes at high cost — both in terms of design complexity as well as numeric complexity of the control law.

As an outlook to possible future research, several other approaches could be attempted in such high-complexity designs. To meet the numeric challenges associated to $\mu$ bounds calculation, especially in the present case where a low number of parameters is repeated often, it seems reasonable to attempt numeric search methods to empirically find improved $\mu$ bounds. Also, $\mu$ computation algorithms without the need of fine frequency gridding could alleviate the encountered difficulties [25].

This study considers only the lateral motion of the BWB aircraft which is decoupled from the longitudinal motion as long as the deviation of the flight mechanic variables remain sufficiently close to trimmed level flight conditions and the linearized system models remain valid. However, even without longitudinal/lateral coupling in the underlying system models, it is important to simulate both dynamics simultaneously in order to verify that control surface deflection/rate limitations are obeyed also in combined maneuvers (such as in coordinated turns). 
In conclusion, the findings of this work underline the importance of efficient LFR modeling for DK-/DGK-iteration-based control design. The encountered challenges demonstrate the need for algorithms which allow to generate efficient LFRs whose parameterization accuracy is optimized for the envisaged control task, for example, through frequency-weighted error minimization.

\section{CONCLUDING REMARKS}

This paper presents results for an incremental robust feedback control design of a lateral inner-loop control law for a large BWB passenger aircraft predesign model. Starting with an initial control law that already provides basic response shaping and flexible mode damping, the main design goal of this work is to further increase the damping of the flexible modes robustly despite the presence of strong parameter-dependent plant variation. The DGK-iteration synthesis procedure is utilized and several LFR formulations of the aircraft model parameter dependency are tested. The highest-complexity attempt involving a high-accuracy parametric LFR cannot be handled computationally. A simple, manual ad-hoc uncertainty formulation leads to quick results with high nominal performance but fails to provide robustness in validation. Finally, a reduced-accuracy parametric LFR is utilized which leads to a computationally demanding design, but yields a control law that robustly stabilizes and attenuates the flexible dynamics above the level provided by the initial control law. High-fidelity validation studies of these control laws via simulations are necessary at a later stage of control design in order to quantify the effects of model uncertainties and errors as well as longitudinal and lateral coupling.

\section{ACKNOWLEDGMENTS}

This work was financially supported by the European Union Framework Programme 7 under the FP7 project No. 213321 [26].

\section{REFERENCES}

1. Hanel, M. 2001. Robust integrated flight and aeroelastic control system design for a large transport aircraft. VDI-Verlag. No. 866.

2. Kron, A., J. de Lafontaine, and D. Alazard. 2003. Robust 2-DOF $H$-infinity controller for highly flexible aircraft: Design methodology and numerical results. Can. Aeronautics Space J. 49:19-29. 
3. Jeanneau, M., J. Lamolie, G. Puyou, and N. Aversa. 2005. Awiators design of multi-objectives control laws. IFAC.

4. Westermayer, C., A. Schirrer, M. Hemedi, M. Kozek, and A. Wildschek. 2009. Robust $\mathcal{H}_{\infty}$ flight and load control of a flexible aircraft using a 2DOF multi-objective design. 2009 CACS Automatic Control Conference (International) Proceedings.

5. Wildschek, A., R. Maier, M. Hromčík, T. Haniš, A. Schirrer, M. Kozek, C. Westermayer, and M. Hemedi. 2009. Hybrid controller for gust load alleviation and ride comfort improvement using direct lift control flaps. 3rd EUCASS Proceedings.

6. Mialon, B., and M. Hepperle, 2005. Flying wing aerodynamics studies at ONERA and DLR. CEAS Katnet Conference on Key Aerodynamic Technologies. Germany.

7. Skogestad, S., and I. Postlethwaite. 1996. Multivariable feedback control analysis and design. John Wiley \& Sons.

8. Zhou, K., J. C. Doyle, and K. Glover. 1996. Robust and optimal control. Prentice Hall.

9. Balas, G., R. Chiang, A. Packard, and M. Safonov 2010. MATLAB Robust Control Toolbox 3, User's Guide. MathWorks.

10. Magni, J.-F. 2002. Robust modal control with a Toolbox for Use with MATLAB. Kluwer Academic.

11. Schirrer, A., C. Westermayer, M. Hemedi, and M. Kozek. 2011. Robust convex lateral feedback control synthesis for a BWB aircraft. 18th IFAC World Congress Preprints. Milano, Italy. 7262-67.

12. Schirrer, A., C. Westermayer, M. Hemedi, and M. Kozek. 2010. A comprehensive robust control design and optimization methodology for complex flexible-structure systems. 18th Mediterranean Conference (International) on Control and Automation. Marrakech, Morocco.

13. Schirrer, A., C. Westermayer, M. Hemedi, and M. Kozek. 2010. LQ-based design of the inner loop lateral control for a large flexible BWB-type aircraft. 2010 IEEE Multi-Conference on Systems and Control. Yokohama, Japan.

14. Schirrer, A., C. Westermayer, M. Hemedi, and M. Kozek. 2010. Robust $\mathcal{H}_{\infty}$ control design parameter optimization via genetic algorithm for lateral control of a BWB type aircraft. IFAC Workshop on Intell. Control Systems. Sinaia, Romania.

15. Schirrer, A., C. Westermayer, M. Hemedi, and M. Kozek. 2011. Multi-model convex design of a scheduled lateral feedforward control law for a large flexible BWB aircraft. 18th IFAC World Congress Preprints. Milano, Italy. 2126-31.

16. Westermayer, C., A. Schirrer, M. Hemedi, and M. Kozek. 2010. Linear parametervarying control of a large blended wing body flexible aircraft. 18th IFAC Symposium on Automatic Control in Aerospace. Nara, Japan.

17. Stroscher, F., Ö. Petersson, and M. Leitner. 2010. Aircraft structural optimization subject to flight loads - application to a wide body commercial aircraft configuration. EASN Workshop (International) on Aerostructures.

18. Schirrer, A., C. Westermayer, M. Hemedi, and M. Kozek. 2012. Inner-loop control design of a scheduled lateral aeroelastic controller for a large flexible BWB aircraft. 13th Mechatronics Forum Conference (International). Linz, Austria.

19. Hecker, S. 2006. Generation of low order LFT representations for robust control applications. Ph.D. Thesis. Technische Universität München. 
20. Gu, D. W., P. H. Petkov, and M. M. Konstantinov. 2005. Robust control design with MATLAB. Springer Verlag.

21. Benatzky, C. 2006. Theoretical and experimental investigation of an active vibration damping concept for metro vehicles. Dissertation. Vienna: Vienna University of Technology.

22. Gawronski, W. 2004. Advanced structural dynamics and active control of structures. New York: Springer.

23. Dehkordi, V. R., and B. Boulet. 2009. Robust controller order reduction. American Control Conference Proceedings. 3083-88.

24. Safonov, M. G., and R.Y. Chiang. 1988. Model reduction for robust control: A Schur relative error method. Int. J. Adaptive Control Signal Proc. 2(4):25972 .

25. Ferreres, G., and J.M. Biannic. 1998. A $\mu$ analysis technique without frequency gridding. American Control Conference Proceedings, IEEE. 4:2294-98.

26. ACFA 2020 Consortium. 2011. Active control of flexible 2020 aircraft (ACFA 2020). EU FP7 Project No. 213321. URL: http://www.acfa2020.eu, retrieved: January 31, 2011. 